\title{
Strain differences and positive reinforcement with shock'
}

GORDON M. HARRINGTON AND GLENN R. KOHLER

STATE COLLEGE OF IOWA

Groups of 6, 6, and 7 IR and INR rats were adapted to test chambers in total darkness for one week, one month, and two months and habituated to operant bar apparatus for 70, 300, and 600 sessions then tested for operant level, shock contingent bar press, and extinction in 150,150, and 350 sessions, all of $16-2 / 3 \mathrm{~min}$. each. Shock proved positively reinforcing and cumulative shock durations were functions of shock intensity and animal reactivity $(p<.001)$. Absolute response levels declined as a decelerated function of adaptation time. Results were interpreted in accord with an arousal and satiation model.

Hebb's (1955) arousal model suggested that any stimulus might function as a reinforcement eliciting either adient or abient responses depending on stimulus intensity and further predicted that this reinforcement effect should be an inverted U-shaped function of intensity. Harrington \& Linder (1962) proposed the use of mild shock as the most appropriate test of this hypothesis and reported reinforcing effects when shock was substituted for another sensory reinforcement. Subsequently it was shown that rats would acquire a shuttle box response for mild shock positive reinforcement (Harrington \& Kohler, 1966). The present study extends these investigations to operant acquisition in a shock contingent bar press situation.

The theory implies that a more reactive $\mathrm{S}$ is one more readily aroused and hence that the inverted U-shaped functions of stimulus intensity will differ with reactivity in location on the stimulus intensity continuum. Specifically, reactive and non-reactive Ss should show inverted $\mathrm{U}$-shaped response functions to varying intensities of shock reinforcement following bar press and the function for non-reactive Ss should be positively displaced with respect to the function for reactive Ss.

Premack \& Collier (1962) observed that short-term pretest adaptation to test apparatus enhanced light contingent bar press and remarked that this was the least understood of their outcomes. It has been suggested (Harrington, 1963; Harrington \& Kohler, 1966) that sensory reinforcement can be interpreted in terms of simultaneous action of arousal and satiation variables. This is interpreted to mean that with adaptation there is satiation on the stimuli present during adaptation. Thus a new sensory reinforcer would show relatively greater effects with increasing satiation on alternative stimulus sources. A further purpose of this study was to explore this hypothesis with prolonged adaptation and habituation particularly since long term adaptation effects seem not to have been studied except in the early environmental deprivation framework.

\section{Method}

Naive rats, 100-120 days old, from the IR (reactive) and INR (nonreactive) stocks of this laboratory were maintained and tested in total darkness. Each rat was tested in the maintenance chamber in 10 equally spaced sessions daily of $1000 \mathrm{sec}$. with a $20 \mathrm{gm}$ retractable bar. Shock to the floor contingent on bar press was provided from a matched impedance ac source (Campbell \& Teghtsoonian, 1958). Automatic program monitors and necropsies provided criteria for discarding data for health or deviation from design conditions.

Experiments 1, 2, and 3 respectively entailed one week, one month, and two months of adaptation with 70,300 , and 600 bar habituation sessions. Test sessions were:

Experiment 1: 50 operant level, $501.2 \mathrm{v}$ shock, and 50 extinction with three IR and three INR Ss.

Experiment 2: 100 operant level and $501.2 \mathrm{v}$ shock with two IR and four INR Ss.

Experiment 3: 70 operant level, $701.2 \mathrm{v}, 704.0 \mathrm{v}$, $700.4 \mathrm{v}$, and 70 extinction with three IR and four INR Ss. Results

Experiment 1. The mean percent cumulative bar press duration for shock reinforcement was $54 \%$ of operant level, a nonsignificant $(t=2.34, p>.05, d f=5)$ decrement. Extinction level was $24 \%$ of operant level, a highly significant decrement $(t=15.5, p<.01, \mathrm{df}=5)$.

Experiment 2. The mean cumulative bar press duration operant level of the second 50 sessions was $41 \%$ of the first 50 sessions following 300 sessions of adaptation. This decrement was significant $(t=3.13, p<.05$, $\mathrm{d} f=5$ ). Shock reinforcement produced a nonsignificant increment over the operant level of the second 50 operant sessions $(t=1.36)$.

Experiment 3. After 600 adaptation sessions inspection of bar habituation data suggested that decrement in response had approached an asymptote. Figure 1 shows that the mean bar press time as a function of operant level was the predicted inverted $U$ for the reactive animals. For non-reactive animals the function reached a maximum at the maximum shock level used. Since it is well known that higher shock levels are aversive it can reasonably be assumed that this was a portion of an inverted $U$.

For statistical test purposes, the alternative hypothesis is composite and posits first interaction between strains and shock level and second, a shock effect within both (joint P) strains. Using percent of operant level, interaction was significant $(F=7.63, \mathrm{df}=4 / 20$, $\mathrm{p}<.001$ ) as was shock effect (reactive $\mathrm{F}=3.92, \mathrm{df}=4 / 8$; non-reactive $\mathrm{F}=7.08, \mathrm{df}=4 / 12$; joint $\mathrm{p}<.001$ ). 


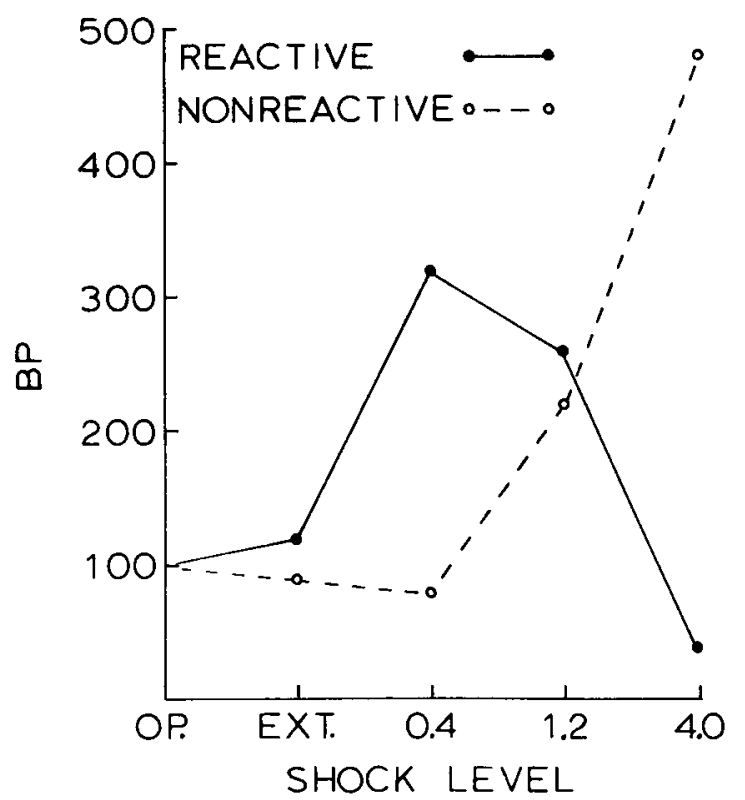

Fig. 1. Cumulative bar press time as per cent of operant time for differing shock levels with zero shock extinction.

Figure 1 also shows that the operant level and extinction level were substantially the same. Thus the decrement found in Experiments 1 and 2 was not found in this situation with more extended adaptation time.

\section{Discussion}

The literature on sensory reinforcement has been concerned largely with light controlled behavior. Lockard (1963) suggested that the effects of light might better be construed under a category of light related behavior. To this end he examined the unique physiologic role of light for rodents and emphasized the importance of duration meisures and influence of prior maintenance conditions. Essentially the same behavioral phenomena that have been found with light reinforcement were found here with shock reinforcement showing broader value in the behavioral category rather than stimulus category approach.
With one week of dark adaptation operant levels were still declining at a very rapid rate. The effect of shock reinforcement seemed to be one of partially counteracting a continuing decremental function. With one month of adaptation operant levels were still declining but shock increment appeared greater than the adaptation decrement. With two months of adaptation no further operant level decrement was observed. Hence one could infer that response level is a negatively accelerated decreasing function of dark maintenance time or adaptation.

These data are consistent with the arousal-satiation model: stimulus reinforcement without association with other variables, reinforcing effect an inverted U-shaped function of stimulus intensity, maximum and operant zeros varying inversely with reactivity, and sensory deprivation or prolonged habituation producing a decelerating decremental effect on operant level but enhancing the relative level of sensorily reinforced behavior.

\section{References}

Campbell, B. A., \& Teghtsoonian, R. Electrical and behavioral effects of different types of shock stimuli on the rat. $J$. comp. physiol. Psychol., 1958, 51, 185-192.

Harrington, G. M, \& Linder, W. K. A positive reinforcing effect of electrical stimulation. J. comp. physiol. Psychol., 1962, 66, 1014-1015.

Harrington, G. M. Stimulus intensity, stimulus satiation, and optimum stimulation with light-contingent bar-press. Psychol. Rep., $1963,13,107-111$

Harrington, G. M., \& Kohler, G. R. Sensory deprivation and sensory reinforcement with shock. Psychol. Rep., 1966, 18, 802-808.

Hebb, D. O. Drives and the CNS (Conceptual Nervous System). Psychol. Rev., 1955, 62, 243-254.

Lockard, R. B. Some effects of light upon the behavior of rodents. Psychol. Bull., 1963, 60, 509-529.

Premack, D., \& Collier, G. Analysis of nonreinforcement variables affecting response probability. Psychol. Monogr., 1962, 76, No. 5 (Whole No. 524).

\section{Note}

1. This research was supported by the U. S. Public Health Service M-6491 (A), the National Science Foundation, G22137, and the State College of Iowa, 302-40. 